\title{
Complementarity of indirect and accelerator dark matter searches
}

\author{
G. Bertone, ${ }^{1}$ D. G. Cerdeño, ${ }^{2}$ M. Fornasa,${ }^{3}$ L. Pieri, ${ }^{4,5}$ R. Ruiz de Austri, ${ }^{6}$ and R. Trotta ${ }^{7}$ \\ ${ }^{1}$ GRAPPA Institute, University of Amsterdam, Science Park 904, 1090 GL Amsterdam, Netherlands \\ ${ }^{2}$ Departamento de Física Teórica, and Instituto de Física Teórica UAM/CSIC, Universidad Autónoma de Madrid, \\ Cantoblanco, E-28049 Madrid, Spain \\ ${ }^{3}$ Instituto de Astrofísica de Andalucía (CSIC), E-18008, Granada, Spain \\ ${ }^{4}$ Dipartimento di Fisica, Universitá di Trento, Via Sommarive 14, I-38123, Trento, Italy \\ ${ }^{5}$ Istituto Nazionale di Fisica Nucleare, sede di Padova, Via Marzolo 8, 35131 Padova, Italy \\ ${ }^{6}$ Instituto de Física Corpuscular, IFIC-UV/CSIC, Valencia, Spain \\ ${ }^{7}$ Astrophysics Group, Imperial College London, Blackett Laboratory, Prince Consort Road, London SW7 2AZ, United Kingdom
}

(Received 27 November 2011; published 19 March 2012)

Even if supersymmetric particles are found at the Large Hadron Collider (LHC), it will be difficult to prove that they constitute the bulk of the dark matter (DM) in the Universe using LHC data alone. We study the complementarity of LHC and DM indirect searches, working out explicitly the reconstruction of the DM properties for a specific benchmark model in the coannihilation region of a 24-parameters supersymmetric model. Combining mock high-luminosity LHC data with presentday null searches for gamma rays from dwarf galaxies with the Fermi Large Area Telescope, we show that current Fermi Large Area Telescope limits already have the capability of ruling out a spurious wino-like solution which would survive using LHC data only, thus leading to the correct identification of the cosmological solution. We also demonstrate that upcoming Planck constraints on the reionization history will have a similar constraining power and discuss the impact of a possible detection of gamma rays from DM annihilation in the Draco dwarf galaxy with a Cherenkov-Telescope-Array-like experiment. Our results indicate that indirect searches can be strongly complementary to the LHC in identifying the DM particles, even when astrophysical uncertainties are taken into account.

DOI: 10.1103/PhysRevD.85.055014

PACS numbers: $95.35 .+\mathrm{d}, 12.60 . \mathrm{Jv}$

\section{INTRODUCTION}

One of the most important questions in dark matter research $(\mathrm{DM})$ is to identify a possible DM candidate seen at the Large Hadron Collider (LHC) with the particle responsible for the cosmological relic density. Some of us have recently shown [1] (hereafter "Paper I") that a convincing identification of DM particles [2-6] can be achieved with a combined analysis of direct-detection and accelerator data. The starting point of Paper I was the simulated response of the LHC to a specific benchmark in the coannihilation region of a 24-parameters minimal supersymmetric standard model (MSSM) and the attempt to reconstruct the properties of the DM particle, in this case the Supersymmetric neutralino, using only these simulated data. As already discussed in Ref. [7], even $300 \mathrm{fb}^{-1}$ of LHC data with a center-of-mass energy of $14 \mathrm{TeV}$ (i.e. with a data set that should only become available by 2018 or so, based on current plans) would not allow us to identify the neutralino as the sole constituent of the DM in the Universe. Assuming a standard expansion rate at freezeout, in fact, the posterior probability obtained after imposing the simulated LHC data exhibits multiple modes, corresponding to different neutralino compositions, and a broad peak around the true solution.

We argued in Paper I that a robust and powerful way of breaking the degeneracy in the parameter space, and therefore of identifying the DM particle, is to combine accelerator data with ton-scale direct-detection experiments measuring the recoil energy of nuclei struck by DM particles (see Refs. [3-5] and references therein), which should become available over a similar time scale. We demonstrated that a simple ansatz on the local density of DM particles (which assumes that the relative abundance of the particles discovered in accelerators with respect to DM is the same locally as their relic density in the Universe) is sufficient to eliminate the spurious mode in the posterior distribution and to constrain the DM properties around the true benchmark value. Similarly to the case of accelerators, a single (realistic) direct-detection experiment can hardly provide an accurate determination of the DM properties $[8,9]$, but a second direct detection with a different target would actually allow a much more precise determination of the weakly interacting massive particle (WIMP) mass [10], and if the new target is sensitive to the spin-dependent contribution of the WIMP-nucleus cross section, it could even be used to discriminate among WIMP candidates [11].

Here, we investigate the impact of adding information from indirect, instead of direct, DM searches. This detection strategy is based on the search for the annihilation or decay products of DM particles, such as high-energy photons, neutrinos, and antimatter [4,5]. There are advantages and disadvantages with respect to direct searches. The most obvious advantage is that indirect searches do not 
require dedicated experiments. Although DM physics has historically played a role in establishing the physics case of experiments such as the Fermi Large Area Telescope (LAT) [12,13], HESS [14,15], and IceCube [16,17], there is a broad range of other astrophysical motivations that made the construction of these experiments possible. Among the disadvantages, the biggest one arises from the large uncertainties in the predicted annihilation rates (which in turn are a consequence of our poor knowledge of the distribution of DM in the Galaxy and in other astrophysical structures), as well as in the astrophysical backgrounds (see also the discussion in Ref. [6]).

Gamma rays are often considered ideal messengers for indirect-detection studies, since they are not significantly affected by diffusion or energy losses in the local Universe. The most promising targets are the Galactic center (GC) [18-23] and substructures in the Milky Way halo, including dwarf galaxies [24-31] and intermediate-mass black holes [32-39].

Although it has long been considered as the optimal target, the Galactic center is actually a very problematic region. The first big obstacle to a reliable identification of $\mathrm{DM}$, or at least for the derivation of robust upper limits on the annihilation flux, is represented by the large uncertainties on the DM distribution in a region which is largely dominated by baryons [40-44]. Furthermore, there are strong and poorly understood astrophysical backgrounds at the $\mathrm{GC}$ which significantly complicate the extraction of a DM signal (see also the discussion in Ref. [45-47]). The complementarity of colliders and the indirect DM search towards the GC has already been considered in Ref. [48]. As for intermediate-mass black holes, the formation scenarios are not very predictive and rather uncertain; therefore, although they might be discovered as a class of objects with an identical gamma-ray spectrum and no astrophysical counterparts, the nondetection does not set stringent constraints on the DM particles.

Here, we focus instead on dwarf spheroidal galaxies (dSphs) and analyze the dramatic implications for the reconstruction procedure of DM properties from future LHC data combined with the current Fermi LAT combined analysis of 10 dwarfs [49]. We demonstrate that the spurious solution at low relic density is ruled out by Fermi LAT, if one assumes that the particle found at the LHC makes up all of the DM in the Universe. We also discuss the constraints that should become available in the next few years with the upcoming ground-based gamma-ray experiment Cherenkov Telescope Array (CTA), and with a suitable analysis [50-54] of Planck satellite data.

The paper is organized as follows: in Sec. II, we describe our theoretical setup and the implementation of experimental constraints. In Sec. III, we show how the constraining power of future LHC data can be improved by including current Fermi LAT constraints on the gammaray flux from dwarf galaxies, future Planck data probing the reionization history of the Universe, and a possible detection of gamma rays from Draco with CTA. We conclude in Sec. IV.

\section{THEORETICAL SETUP}

\section{A. Benchmark model}

For concreteness, we will start by defining our theoretical framework. In this work, we will consider that the MSSM is the correct description for physics beyond the standard model and that it also provides a solution to the DM problem in terms of the lightest neutralino, $\tilde{\chi}_{1}^{0}$.

Neutralinos are physical superpositions of the superpartners of the $B$ and $W$ gauge bosons (bino and wino, respectively) and Higgs bosons (Higgsinos), and their phenomenological properties are extremely sensitive to their specific composition. In particular, their annihilation cross section in the early Universe (which determines their relic abundance) as well as in DM haloes (which affects the gamma-ray flux that can be observed in indirect DM detection) has an uncertainty of several orders of magnitude depending on the neutralino composition. It is worth remembering in this sense that neutralinos with a large wino or Higgsino component are known to have a larger annihilation cross section (and hence a smaller relic density) than those in which the bino component dominates. For this reason, obtaining the neutralino composition in the LHC is a key ingredient for being able to determine whether or not this particle is the main component of the DM.

We follow here the theoretical setup and the notation in Paper I: we adopt a minimal supersymmetric extension of the standard model [called phenomenological MSSM (pMSSM)] with 24 free parameters, corresponding to its CP-conserving version. The input parameters in the MSSM Lagrangian are the following: coefficents of the trilinear terms for the three generations $\left(A_{\tau}, A_{b}\right.$, and $\left.A_{t}\right)$; the mass terms for gauginos $\left(M_{1}, M_{2}\right.$, and $\left.M_{3}\right)$, for which no universality assumption is made; right-handed and lefthanded squarks and leptons masses $\left(m_{Q_{i}}^{2}, m_{u_{i}}^{2}, m_{d_{i}}^{2}, m_{L_{i}}^{2}\right.$, $m_{e_{i}}^{2}$ with $i=1,2$ and 3 ); the mass of the pseudoscalar Higgs $\left(m_{A}\right)$; the Higgsino mass parameter $(\mu)$; and finally the ratio of the vacuum expectation values of the two Higgs bosons $(\tan \beta)$. We assume a benchmark point corresponding to the low-energy extrapolation of model LCC3 as defined in Ref. [7]. This benchmark is representative of supersymmetric (SUSY) models in the coannihilation region, where the lightest neutralino is almost degenerate in mass with the lightest stau. In this region, coannihilation effects reduce the neutralino relic abundance down to values compatible with the results from the WMAP satellite [55], and therefore, the mass difference between the neutralino and the lightest stau is a fundamental parameter for the reconstruction of the relic density. 
For this MSSM model, the structure of the neutralino sector is very characteristic of the MSSM, with the lightest neutralino being binolike, the second-lightest neutralino being winolike, and the two heavier neutralinos corresponding to Higgsino-like states with a relatively large mass (approximately $460 \mathrm{GeV}$ ). This structure is a consequence of the low-energy hierarchy $M_{1}<M_{2}<\mu$ among the gaugino and Higgsino mass parameters at low energy. Because of this, the lightest chargino is also winolike, the heaviest corresponding to the charged Higgsino.

\section{B. Statistical analysis}

In order to constrain the parameters $\mathbf{x}$ described above of our 24-dimensional SUSY model, we make use of Bayes' theorem:

$$
p(\mathbf{x} \mid \mathbf{d})=\frac{p(\mathbf{d} \mid \mathbf{x}) p(\mathbf{x})}{p(\mathbf{d})},
$$

which updates the so-called prior probability density $p(\mathbf{x})$, representing the (lack of) knowledge on the 24-dimensional space before taking into account the experimental constraints, d, to the posterior probability function (PDF) $p(\mathbf{x} \mid \mathbf{d})$. The latter is the probability density after the data have been taken into account via the likelihood function, $p(\mathbf{d} \mid \mathbf{x})=\mathcal{L}(\mathbf{x})$. Furthermore, on the right-hand side of Eq. (1), $p(\mathbf{d})$ is the Bayesian evidence which, in our case, can be dropped since it simply plays the role of a normalization constant for the posterior in this context (see Ref. [56] for further details).

The posterior encodes both the information contained in the priors and in the experimental constraints, but, ideally, it should be largely independent of the choice of priors, so that the posterior inference is dominated by the data contained in the likelihood. If some residual dependence on the prior $p(\mathbf{x})$ remains, this should be considered as a sign that the experimental data employed are not constraining enough to override completely different plausible prior choices, and therefore the resulting posterior should be interpreted with some care, as it might depend on the prior assumptions $[57,58]$. For the practical implementation of the Bayesian analysis sketched above, we employed the SUPERBAYES code $[59,60]$, extending the publicly available version 1.5 to the 24 dimensions of our SUSY parameter space. To scan in an efficient way the SUSY parameter space, we have upgraded the MULTINEST [61] algorithm included in SUPERBAYES to the latest MULTINEST release (version 2.7), with uniform priors on the pMSSM parameters as in Paper I, to which the reader is referred for full details.

The set of LHC simulated measurements that we use as constraints in our analysis corresponds to that in Table 6 of Ref. [7], which assumes an integrated luminosity of $300 \mathrm{fb}^{-1}$. Furthermore, as pointed out in Ref. [62], the neutralino-stau mass difference can be measured with an accuracy of $20 \%$ with a luminosity of $10 \mathrm{fb}^{-1}$ in models
TABLE I. Sparticle spectrum (in $\mathrm{GeV}$ ) for our benchmark SUSY point and relative estimated measurements errors at the LHC (standard deviation $\sigma$ ).

\begin{tabular}{lcc}
\hline \hline Mass & Benchmark value, $\mu$ & LHC error, $\sigma$ \\
\hline$m\left(\tilde{\chi}_{1}^{0}\right)$ & 139.3 & 14.0 \\
$m\left(\tilde{\chi}_{2}^{0}\right)$ & 269.4 & 41.0 \\
$m\left(\tilde{e}_{1}\right)$ & 257.3 & 50.0 \\
$m\left(\tilde{\mu}_{1}\right)$ & 257.2 & 50.0 \\
$m(h)$ & 118.50 & 0.25 \\
$m(A)$ & 432.4 & 1.5 \\
$m\left(\tilde{\tau}_{1}\right)-m\left(\tilde{\chi}_{1}^{0}\right)$ & 16.4 & 2.0 \\
$m\left(\tilde{u}_{R}\right)$ & 859.4 & 78.0 \\
$m\left(\tilde{d}_{R}\right)$ & 882.5 & 78.0 \\
$m\left(\tilde{s}_{R}\right)$ & 882.5 & 78.0 \\
$m\left(\tilde{c}_{R}\right)$ & 859.4 & 78.0 \\
$m\left(\tilde{u}_{L}\right)$ & 876.6 & 121.0 \\
$m\left(\tilde{d}_{L}\right)$ & 884.6 & 121.0 \\
$m\left(\tilde{s}_{L}\right)$ & 884.6 & 121.0 \\
$m\left(\tilde{c}_{L}\right)$ & 876.6 & 121.0 \\
$m\left(\tilde{b}_{1}\right)$ & 745.1 & 35.0 \\
$m\left(\tilde{b}_{2}\right)$ & 800.7 & 74.0 \\
$m\left(\tilde{t}_{1}\right)$ & 624.9 & 315.0 \\
$m(\tilde{g})$ & 894.6 & 171.0 \\
$m\left(\tilde{e}_{2}\right)$ & 328.9 & 50.0 \\
$m\left(\tilde{\mu}_{2}\right)$ & 328.8 & 50.0 \\
\hline \hline
\end{tabular}

where the squark masses are much larger than those of the lightest chargino and second-lightest neutralino, as is our case. We therefore also include a measurement of the neutralino-stau mass difference in our likelihood. We summarize in Table I the set of LHC measurements adopted in our likelihood. Each of the constraints listed in Table I is

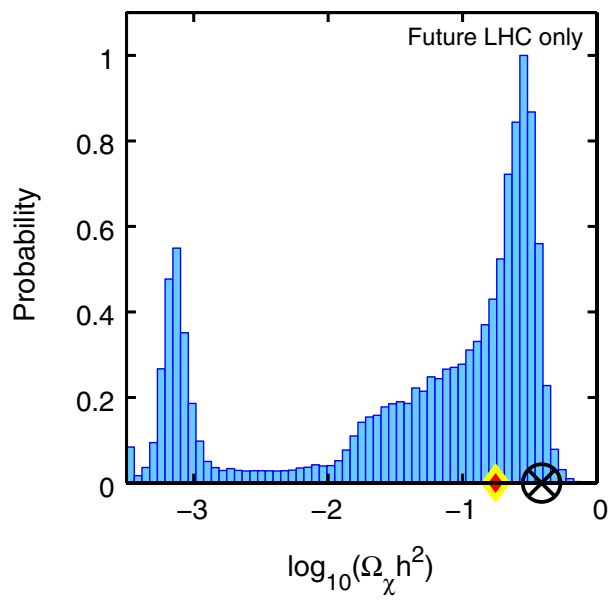

FIG. 1 (color online). 1-dimensional marginalized posterior probability density function for the neutralino relic abundance after LHC hypothetical measurements (given in Table I) are taken as experimental constraints. The best fit is given by the encircled black cross, while the true value is indicated by the yellow/red diamond. 

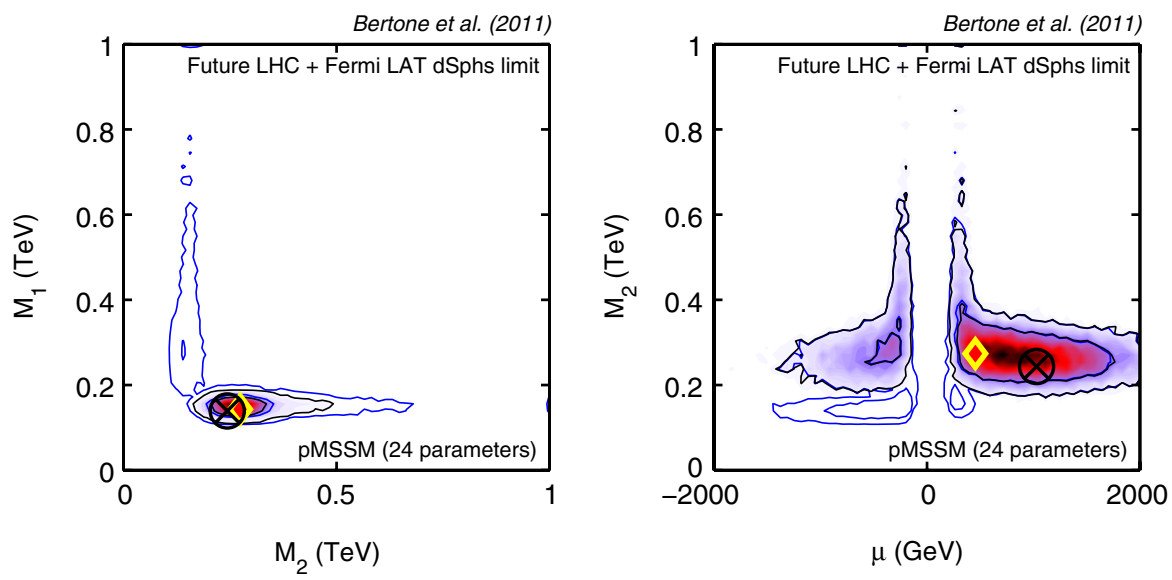

FIG. 2 (color online). 2-dimensional marginalized posterior PDF in the $\left(M_{1}, M_{2}\right)$ and in the $\left(M_{2}, \mu\right)$ planes. The inner and outer contour encloses $68 \%$ and $95 \%$ probability regions, respectively. The blue/empty contours are for the case where LHC-only data are applied, whereas the filled regions with black contours also include current Fermi LAT upper limits from the combined analysis of dSphs. The best fit (for the case with Fermi LAT upper limits) is shown with the encircled black cross, while the true value is given by the yellow/red diamond.

implemented in the likelihood as an independent Gaussiandistributed measurement around the true value $\mu_{i}$ for that observable, with standard deviation $\sigma_{i}$, as given in Table I, i.e. the likelihood from the LHC has the form:

$$
\mathcal{L}_{\mathrm{LHC}}(\mathbf{x}) \propto \prod_{i} \exp \left(-\frac{1}{2} \frac{\left(\mu_{i}-\mu_{i}(\mathbf{x})\right)^{2}}{\sigma_{i}^{2}}\right),
$$

where $\mu_{i}(\mathbf{x})$ is the predicted value for the observable at point $\mathbf{x}$ in parameter space.

After imposing these constraints, the one-dimensional marginalized posterior PDF for the neutralino relic abundance is shown in Fig. 1. Despite the good accuracy in the determination of some of the supersymmetric masses (for example, the neutralino mass has a relatively small $10 \%$ uncertainty) and especially the mass difference between the neutralino and the stau next-to-lightest supersymmetric particle (crucial to quantify the coannihilation effect), the resulting prediction for the relic abundance spans 4 orders of magnitude. This result clearly illustrates how the LHC might be unable to quantify the neutralino relic abundance and thus to determine whether or not it is the main ingredient of the DM. Furthermore, the posterior PDF shows a multimodal structure, with two fairly well-separated probability density peaks indicating two physically different solutions.

This uncertainty in the neutralino relic density is a direct consequence of the impossibility of determining its composition in an unambiguous way [7]. Only the two lightest neutralino mass eigenstates are measured (and none of the charginos), which is not enough to constrain the neutralino mass matrix. We illustrate this in Fig. 2, where the posterior PDFs for the parameters in the neutralino mass matrix $\left(M_{1}, M_{2}\right.$, and $\left.\mu\right)$ obtained with LHC-only data are shown by the empty contours. As we can observe, there are two possible solutions that satisfy the LHC constraints: one in which the neutralino is mostly binolike $\left(M_{1}<M_{2}<\mu\right)$ and another one in which it is mostly winolike $\left(M_{2}<M_{1}<\mu\right)$. Moreover, the $\mu$ parameter is not well-determined (since the heavier mass eigenstates are not measured) and varies in a wide range. ${ }^{1}$ This implies that the Higgsino composition of the lightest neutralino can vary significantly.

From the discussion above, it is easy to identify which is the neutralino composition associated to the different solutions of the relic density in the PDF. The peak with $\Omega_{\tilde{\chi}_{1}^{0}} h^{2} \geq 0.1$ in Fig. 1 corresponds to points in the parameter space in which the neutralino is binolike (thus having a smaller annihilation cross section and, consequently, a larger relic abundance, compatible with the relic density measured with cosmological data). The long tail originating in this maximum and extending towards smaller values of $\Omega_{\tilde{\chi}_{1}^{0}} h^{2}$ is obtained for neutralinos with an increasing value of the Higgsino component (that is, those for which the $\mu$ parameter is smaller in Fig. 2). Finally, the second peak situated at $\Omega_{\tilde{\chi}_{1}^{0}} h^{2} \approx 10^{-3}$ corresponds to winolike neutralinos, which annihilate very efficiently in the early Universe.

\section{INDIRECT DETECTION CONSTRAINTS}

The indirect-detection signals discussed here [gamma rays from dwarfs and modifications of cosmic microwave background $(\mathrm{CMB})$ spectrum] depend on the neutralino

\footnotetext{
${ }^{1}$ Notice that although, in theory, we could have also obtained a solution in which the second-lightest neutralino was Higgsinolike (that is, $\mu<M_{2}$ ), this possibility is constrained by the determination of masses in the Higgs sector, since a light pseudoscalar would have also been present.
} 
self-annihilation cross section $(\sigma v)$ on the neutralino mass, $m_{\tilde{\chi}_{1}^{0}}$, and on the spectrum of standard model particles produced in the annihilation of neutralinos, which enters in the calculation of the total photon spectrum per annihilation (relevant for the search of gamma rays from dwarfs) and of the fraction of energy that couples with the gas during recombination (relevant for $\mathrm{CMB}$ constraints). Indirect searches can therefore be used to constrain these parameters, under specific assumptions on astrophysical quantities, and as we shall see, they allow us to exclude portions of the phenomenological parameter space that would remain viable under future high-luminosity LHC measurements.

\section{A. Fermi LAT constraints from dwarf spheroidal galaxies}

The first 24 months of data obtained by the Fermi LAT telescope in survey mode have been analyzed in Ref. [49] to search for gamma-ray emission from the position of 10 dShps including Draco. The lack of detection allowed us to set constraints on the gamma-ray emission from each dSph and, assuming a certain DM content, on the DM selfannihilation cross section $(\sigma v)$. The gamma-ray flux at energy $E_{\gamma}$ due to DM annihilations from the direction $\Psi$ is given by

$$
\frac{d \Phi_{\gamma}}{d E_{\gamma}}\left(E_{\gamma}, \Psi\right)=\frac{1}{4 \pi} \frac{(\sigma v)}{2 m_{\tilde{\chi}_{1}^{0}}^{2}} \frac{d N_{\gamma}}{d E_{\gamma}} \int_{\Delta \Omega} \int \frac{\rho^{2}(r(s, \Psi))}{s^{2}} d s d \Omega,
$$

where $d N_{\gamma} / d E_{\gamma}=\sum_{f} d N_{\gamma}^{f} / d E_{\gamma}$ is the total differential photon spectrum per annihilation, obtained by adding up the contributions of all annihilation channels $f$, weighted by the corresponding branching ratio $B_{f}$. The DM distribution in the dwarf galaxies $\rho(r)$ is assumed to be spherically symmetric, and therefore, it is a function only of the radius $r$, which can itself be expressed as a function of the distance along the line-of-sight from the observer $s$, and the angle with respect to the center of the dwarf $\Psi$. To obtain the annihilation rate, the square of the DM density is then integrated along the line-of-sight $s$ over the solid angle $\Delta \Omega$.

If no excess emission is detected from the direction of a dwarf (which is the case so far), then Eq. (3) allows one to translate an upper limit in flux into an upper limit on the DM parameters, once a specific DM profile is assumed for the dSph. In Ref. [49], the Fermi LAT collaboration combined the data from $10 \mathrm{dSphs}$ into a single likelihood analysis, obtaining an upper limit on $(\sigma v)$ of the order of $10^{-25} \mathrm{~cm}^{3} \mathrm{~s}^{-1}$ for a DM mass around $130 \mathrm{GeV}$ (in the case of annihilation into $b \bar{b}$ ). The analysis in Ref. [49] accounts for the astrophysical uncertainties on the DM profile for each dSph. The DM profile can be determined from kinematic data of the member stars, and, in particular, measurements of stellar velocity dispersion can be used to build a likelihood function that depends on the parameters defining a DM halo profile [31]. These quantities have then been included in the likelihood analysis of Fermi LAT data, so that their final result accounts for our relatively poor knowledge of DM in dSphs (see Ref. [49] for more details).

We focus here for definitiveness on the upper limit on $(\sigma v)$ derived for a DM particle annihilating to $b$ quarks, taken from Fig. 2 of Ref. [49]. That same figure also indicates how the upper limit depends on the dominant annihilation channel. With a neutralino mass around $130 \mathrm{GeV}$, as is the case for our benchmark point, the constraints are all within a factor of 2 from the case of annihilation into $b$ quarks, and this is not enough to change our results significantly (see later). The only exception is for an annihilation predominantly into $\mu^{+} \mu^{-}$, for which the Fermi LAT upper limit is approximately an order of magnitude weaker. However, we checked that the branching ratio into muons is subdominant (with a branching ratio smaller than about 0.1 ) for all the samples in our scan, once LHC data are included; hence, this case can be discounted.

We include the information provided by Fermi LAT on the combined analysis of the $10 \mathrm{dSphs}$ by assigning a likelihood of 0 to all samples in our LHC-only scans that have an annihilation cross section larger than the 95\% upper limit in Fig. 2 of Ref. [49]. A more detailed analysis would include the full likelihood function in a more refined way, but this is not necessary for the purpose of our study. We stress that in order to implement the Fermi LAT constraints, we make the assumption that the neutralino found at the LHC contributes $100 \%$ to the nonbaryonic DM in dwarf galaxies, similarly to the consistency check approach of Paper I. The resulting two-dimensional posterior PDF in the planes $\left(m_{\chi}, \sigma v\right)$ and $\left(\Omega_{\chi} h^{2}, \sigma_{\chi-p}^{\mathrm{SI}}\right)$ are shown in the first column of Fig. 3 (filled contours), where they are compared with the case where LHC-only future contraints are used (blue/emtpy contours). Focusing first on the top panel, we notice that the LHC alone (empty contours) is not going to be able to identify the correct mode in the posterior distribution. The secondary mode, at large values of $\sigma v$, corresponds to the case where the neutralino is winolike. This solution can, however, be ruled out once the Fermi LAT information from dSphs is included in the likelihood (filled contours). The resulting identification of the correct cosmological solution in terms of the predicted relic density is confirmed by the plot in the second panel, showing how Fermi LAT limits can eliminate the mode in the distribution corresponding to subdominant relic density. The last row of Fig. 3 shows the one-dimensional marginalized PDF for the relic density, comparing the LHC-only constraints (empty histogram), given in Fig. 1, with what can be obtained by combining LHC with Fermi LAT. We see clearly that the winolike neutralino solution disappears in this latter case.

The impact of Fermi LAT data can also be observed in the reconstruction of the neutralino mass parameters of 

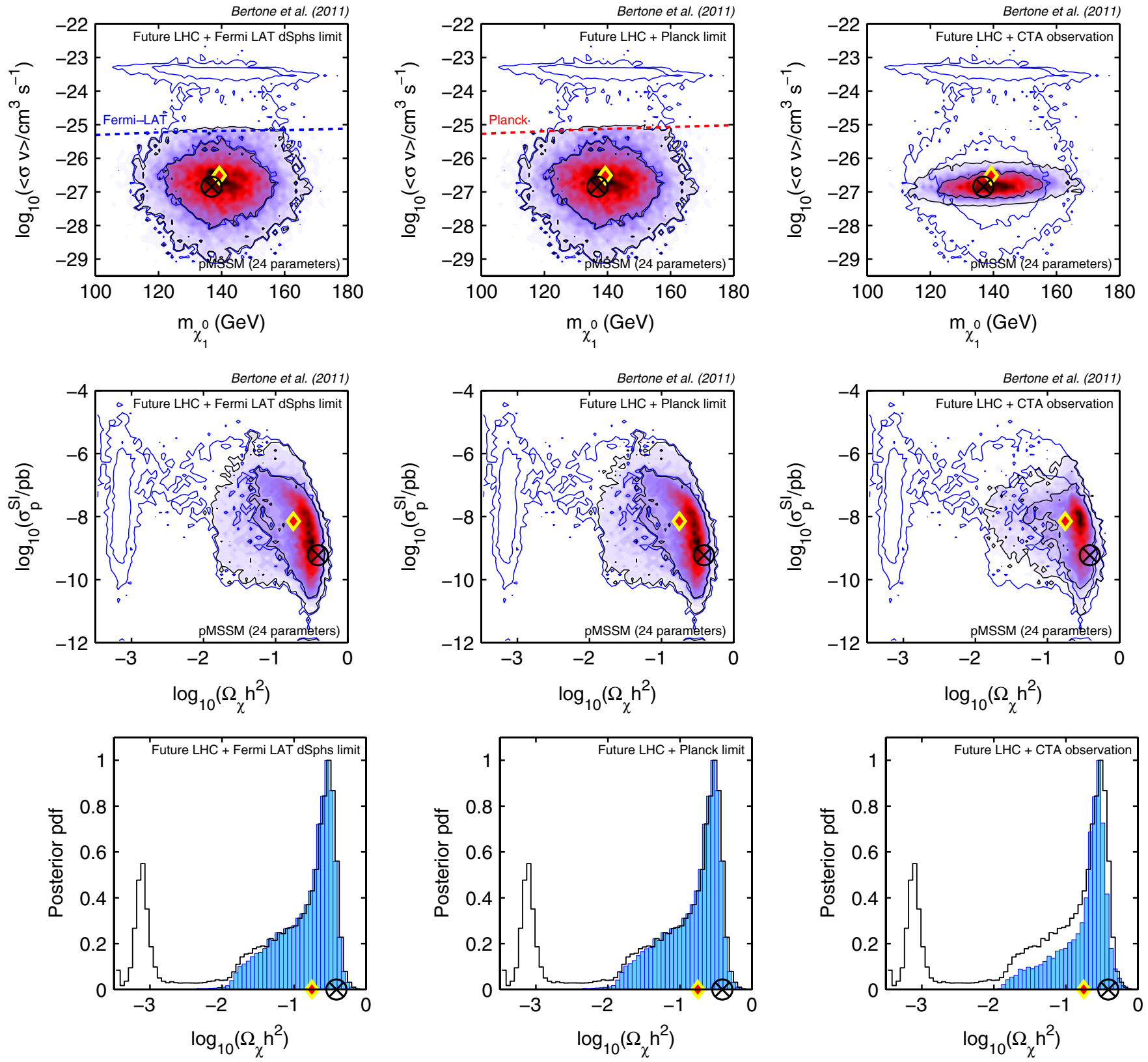

FIG. 3 (color online). 2-dimensional marginal posterior PDF in the plane $\left(m_{\chi},(\sigma v)\right)$ (top row) and $\left(\Omega_{\chi} h^{2}, \sigma_{\chi}^{\text {SI }}\right)$ (middle row), including simulated future LHC data only (blue/empty contours) and adding the Fermi LAT upper limit from the combined analysis of dSphs (left column, filled regions). The second columns combines LHC data with the upper limit expected from Planck on the reionization of the CMB radiation, while the third column combines LHC data with a hypothetical detection of gamma rays from the Draco dSph obtained with CTA. The bottom row shows the 1-dimensional marginal PDF for the relic density $\Omega_{\chi} h^{2}$. The inner and outer contour encloses $68 \%$ and $95 \%$ probability regions, respectively. The best fit is shown with the encircled black cross while the true value is given by the yellow/red diamond. The black continuous line in the bottom row indicates the PDF of $\Omega_{\chi} h^{2}$ for the LHC-only case.

Fig. 2, where Fermi can rule out the region where $M_{2}<$ $M_{1}$. However, Fermi has little impact in the reconstruction of the $\mu$ parameter, and therefore the Higgsino composition of the neutralino is not well determined. As a consequence, the reconstruction of the neutralino relic density (bottom panel of Fig. 3) still displays the characteristic long tail towards small values of $\Omega_{\tilde{\chi}_{1}^{0}} h^{2}$. In practice, even the combined data can only constrain the relic density within an order of magnitude or so of the true value.

\section{B. Planck constraints from recombination history}

The most robust constraints on the annihilation cross section are perhaps those arising from observations of the CMB radiation [50-54], since they do not depend on 
poorly known quantities such as the DM profile at the Galactic center or in dwarf galaxies.

The annihilation of DM particles around redshift $\sim 1000$ inevitably affects the processes of recombination and reionization, modifying the evolution of the free electron fraction $x_{e}$ and the temperature of baryons. The evolution of the ionization fraction $x_{e}$ satisfies the following equation:

$$
\frac{d x_{e}}{d z}=\frac{1}{(1+z) H(z)}\left[R_{s}(z)-I_{s}(z)-I_{X}(z)\right]
$$

where $R_{S}$ is the standard recombination rate [63,64], $I_{s}$ the ionization rate by standard sources, and the $I_{X}$ term represents a "nonstandard" source of ionization; in our case, it takes into account that, during recombination, annihilations of DM particles increase the ionization rate both by direct ionization from the ground state and by contributing additional Lyman- $\alpha$ photons. Therefore, the ionization rate due to DM annihilations can be written as

$$
I_{X}(z)=I_{X i}(z)+I_{X \alpha}(z),
$$

where $I_{X i}$ is the ionization rate due to ionizing photons, and $I_{X \alpha}$ is the ionization rate due to additional Lyman- $\alpha$ photons. The rate of energy release $d E(z) / d t$ per unit of volume by a relic self-annihilating DM particle at redshift $z$ is given by

$$
\frac{d E}{d t}(z)=\rho_{c}^{2} c^{2} \Omega_{\mathrm{DM}}^{2}(1+z)^{6} f \frac{\sigma v}{m_{\chi}} .
$$

Here, $\Omega_{\mathrm{DM}}$ is the DM density parameter, and $\rho_{c}$ is the critical density of the Universe today; the parameter $f$ indicates the fraction of energy which is absorbed overall by the gas, under the approximation that the energy absorption takes place locally. The latter parameter, together with the DM mass and annihilation cross section, define the parameter we call $p_{\text {ann }} \equiv f \sigma v / m_{\chi}$.

Both the nonstandard ionization rates $I_{X i}$ and $I_{X \alpha}$ are related to the energy release rate as follows:

$$
I_{X i}=\frac{C \chi_{i}}{n_{H}(z) E_{i}} \frac{d E}{d t}(z), \quad I_{X \alpha}=\frac{(1-C) \chi_{\alpha}}{n_{H}(z) E_{\alpha}} \frac{d E}{d t}(z)
$$

where $E_{i}$ is the average ionization energy per baryon, $E_{\alpha}$ is the difference in binding energy between the $1 s$ and $2 p$ energy levels of a hydrogen atom, $n_{H}(z)$ is the number density of hydrogen nuclei, and $\chi_{i}=\chi_{\alpha}=\left(1-x_{e}\right) / 3$ are the fractions of energy going to ionization and to Lyman- $\alpha$ photons, respectively. A fraction of the energy released by annihilating DM particles goes into heating of baryonic gas, adding an extra term in the standard equation for the evolution of the matter temperature (see Eqs. (9) and (10) of Ref. [50]).

In Ref. [50], the angular power spectrum of CMB anisotropies was computed, taking into account the presence of DM annihilations, and an upper limit of $2.0 \times$ $10^{-6} \mathrm{~m}^{3} \mathrm{~s}^{-1} \mathrm{~kg}^{-1}$ was derived from WMAP5 data at the 95\% confidence level. This limit was already sufficient to exclude models that exhibit a large Sommerfeld enhancement for $(\sigma v)$, such as those proposed to fit the PAMELA and ATIC results. The upper limit is expected to become an order of magnitude more stringent, of order $1.5 \times$ $10^{-7} \mathrm{~m}^{3} \mathrm{~s}^{-1} \mathrm{~kg}^{-1}$, with upcoming Planck data. In order to study the impact of such data on LHC constraints on the pMSSM parameters, we have added a projected upper limit on $p_{\text {ann }}$ from Planck, shown as the dashed red line in the top central panel of Fig. 3. In doing so, we adopted a fixed value $f=0.5$ for the fraction of energy absorbed by the gas. Although the actual value of $f$ depends on the particle physics characteristics of the neutralino, detailed estimations of $f$ (e.g., Ref. [51]) show that this is a reasonable choice.

As shown in the central panels of Fig. 3, we find that future Planck constraints will complement LHC data in a very similar way to those arising from the nondetection of gamma rays from Fermi LAT. The combination of LHC and Planck will exclude regions of the parameter space with a high annihilation cross section and low relic density, leading again to the identification of the correct cosmological solution for the relic density within an order of magnitude (bottom panel).

\section{Constraints from a CTA-like experiment}

We now move on to analyze the implications that an actual detection of excess photons would have on the parameter space of SUSY DM discovered at the LHC. Cherenkov telescopes represent the future (or at least the near future) of gamma-ray experiments. Cherenkov telescopes detect gamma rays indirectly, through the detection of the electromagnetic shower produced by the interaction of primary gamma rays with the atmosphere. The shape of the image created by the shower in the telescope camera allows us to discriminate photons from hadrons, and it also provides information on the incident gamma ray as, e.g. energy and direction (see Ref. [65] for a recent review on the Cherenkov telescopes and their role in DM searches). We focus here on the upcoming CTA [66]. Current plans are to build a facility on two sites, one on each hemisphere, with telescopes of three different sizes, for a total of about 100 instruments. Such a large number of telescopes, combined with a large field of view, will allow one to monitor more sources at once and also to perform surveys of large portions of the sky $[67,68]$. CTA sensitivity is estimated to go down by a factor of 10 with respect to current Cherenkov telescopes, and the energy range will cover the interval from $10 \mathrm{GeV}$ to $200 \mathrm{TeV}[66,69]$. The CTA collaboration will complete its design phase in 2 years from now. Currently, different prototypes of the final telescopes are already under construction and will be tested. A realistic time scale for the construction of the experiment is 5 years from now.

For our purposes, we will consider a rather optimistic energy threshold of $20 \mathrm{GeV}$. Our benchmark point 
corresponds to a neutralino mass of $139.3 \mathrm{GeV}$ (see Table I), so gamma rays from DM annihilation are expected to fall in an energy range for which a reasonable estimation of CTA effective is $10^{4} \mathrm{~m}^{2}$ (contrary to the largest values predicted for higher energies).

We consider CTA observations of Draco, and we assume for its astrophysical factor an informative prior $p(\lambda)$, modeled as a Gaussian distribution with mean $1.20 \times$ $10^{19} \mathrm{GeV} \mathrm{cm}^{-5} \mathrm{sr}$ and standard deviation of $0.31 \times$ $10^{19} \mathrm{GeV} \mathrm{cm}^{-5} \mathrm{sr}$, following Table 4 of Ref. [70]. With such prescriptions (and for an astrophysical factor equal to the mean of the Gaussian prior), our benchmark SUSY point predicts a flux of $N_{\mathrm{CTA}}=7.1$ photons from the halo of Draco above $20 \mathrm{GeV}$, with 1000 hours of observation. As we are interested in the constraining power of CTA around the true value of the parameters, we neglect Poisson noise in realizing the counts, and we thus assume an observed number of photons above $20 \mathrm{GeV} \hat{N}_{\text {CTA }}=7$. There is not enough statistics to build a gamma-ray spectrum, so we will only consider the information coming from the detection of all photons above the energy threshold. This is implemented in the likelihood as an additional experimental constraint of the following form:

$$
\mathcal{L}_{\mathrm{CTA}}(\mathbf{x})=\int p\left(\lambda N(\mathbf{x}) \mid \hat{N}_{\mathrm{CTA}}\right) p(\lambda) d \lambda,
$$

where $N(\mathbf{x})$ is the predicted number of photons above threshold for a pMSSM parameters value $\mathbf{x}$ and an astrophysical factor equal to unity, $\lambda$ is the unknown true astrophysical factor for Draco (which we marginalize over), $p\left(\lambda N(\mathbf{x}) \mid \hat{N}_{\text {CTA }}\right)$ is a Poisson distribution in $\hat{N}_{\text {CTA }}$, and $p(\lambda)$ represents its prior.

The results of augmenting LHC data with a future CTA-like detection can be seen in the last column of Fig. 3. With a detection of only 7 photons from Draco, the LHC constraints on the DM self-annihilation cross section collapse from 6 orders of magnitude down to about 1 order of magnitude. As a consequence, the relic abundance can again be constrained around its true value. We point out that this dramatic improvement holds true even when, like we do here, astrophysical uncertainties are marginalized over.

\section{CONCLUSIONS}

We have discussed the complementarity of indirect and accelerator DM searches, and we have shown that current upper limits on the DM self-annihilation cross section arising from Fermi LAT constraints on gamma rays from dwarfs are already sufficiently powerful to break degeneracies in the phenomenological parameter space of DM that would be present even in high-luminosity LHC data. Similar results are obtained if we consider the information that Planck will provide on the distortion of the CMB, or if we assume a possible future detection of gamma rays from Draco by the CTA telescope.
In Paper I, we performed a similar analysis for a combination of accelerator and direct-detection data and demonstrated how a simple yet powerful scaling ansatz (stating that the local contribution of neutralinos to galactic DM was the same as on average in the Universe) led to the correct identification of the cosmological solution. In this paper, we focused on the power of indirect-detection techniques, which do not require such an ansatz. Instead, we assumed that the neutralino seen in colliders makes up the totality of the DM in the Universe (this is what in Paper I was called "the consistency check" approach). This corresponds to asking the question: can the observed neutralinos make all the DM in the local Universe? This approach allows one to discard solutions corresponding to highannihilation cross sections without making any assumption on the expansion rate of the Universe at freeze-out, therefore bypassing all the difficulties arising from all the effects that might possibly modify it (see Ref. [71] for a recent review).

The removal of the spurious winolike mode which would survive with LHC measurements alone and the identification of the correct cosmological solution can be achieved via various indirect-detection channels: current Fermi LAT limits on the flux from dwarfs are already sufficient to this goal (at least for the benchmark point studied here), but Planck constraints will give similar results when they become available in 2013, while probing very different physics. Furthermore, we have shown that a detection of gamma rays from Draco from CTA can lead to similarly stringent conclusions, even when astrophysical uncertainties are included in the analysis.

This work, therefore, makes the case for a vigorous program of indirect searches, especially in the case where the LHC finds evidence for new physics. We have demonstrated that, in this case, indirect-detection methods can have a crucial role to play, even in the case where the experiments only return upper limits to the signal. Together with the complementarity between LHC and direct detection methods presented in Paper I, our results thus highlight the fundamental importance of a multipronged approach to DM identification.

\section{ACKNOWLEDGMENTS}

R. T. would like to thank the University of Zurich for hospitality. M.F. and D. G.C. would like to thank the Spanish MICINN's Consolider-Ingenio 2010 Programme under grant MultiDark CSD2009-00064. D. G. C. is supported by the Ramón y Cajal program of the Spanish MICINN and also acknowledges support from the MICINN under Grant No. FPA2009-08958, the Community of Madrid under Grant No. HEPHACOS S2009/ESP-1473, and the European Union under the Marie Curie-ITN program PITN-GA-2009-237920. The work of G. B. is supported by the ERC Starting Grant WIMPs Kairos. 
[1] G. Bertone, D. G. Cerdeno, M. Fornasa, R. R. de Austri, and R. Trotta, Phys. Rev. D 82, 055008 (2010).

[2] G. Jungman, M. Kamionkowski, and K. Griest, Phys. Rep. 267, 195 (1996).

[3] C. Muñoz, Int. J. Mod. Phys. A 19, 3093 (2004).

[4] G. Bertone, D. Hooper, and J. Silk, Phys. Rep. 405, 279 (2005).

[5] G. Bertone et al., Particle Dark Matter:Observations, Models and Searches, edited by G. Bertone (Cambridge University Press, Cambridge, England, 2010).

[6] G. Bertone, Nature (London) 468, 389 (2010).

[7] E. A. Baltz, M. Battaglia, M. E. Peskin, and T. Wizansky, Phys. Rev. D 74, 103521 (2006).

[8] A. M. Green, J. Cosmol. Astropart. Phys. 08 (2007) 022.

[9] A. M. Green, J. Cosmol. Astropart. Phys. 07 (2008) 005.

[10] M. Drees and C. L. Shan, J. Cosmol. Astropart. Phys. 06 (2008) 012.

[11] G. Bertone, D. G. Cerdeño, J. I. Collar, and B. C. Odom, Phys. Rev. Lett. 99, 151301 (2007).

[12] http://fermi.gsfc.nasa.gov.

[13] W. B. Atwood et al. (LAT Collaboration), Astrophys. J. 697, 1071 (2009).

[14] http://www.mpi-hd.mpg.de/hfm/HESS/pages/about/ telescopes/.

[15] F. A. Aharonian, H. J. Volk, and D. Horns, AIP Conf. Proc. 745, 802 (2005)

[16] http://icecube.wisc.edu/.

[17] F. Halzen and S. R. Klein, Rev. Sci. Instrum. 81, 081101 (2010).

[18] D. Hooper and L. Goodenough, Phys. Lett. B 697, 412 (2011).

[19] T. E. Jeltema and S. Profumo, J. Cosmol. Astropart. Phys. 11 (2008) 003.

[20] M. Regis and P. Ullio, Phys. Rev. D 78, 043505 (2008).

[21] G. Bertone, G. Sigl, and J. Silk, Mon. Not. R. Astron. Soc. 337, 98 (2002).

[22] P. Gondolo and J. Silk, Phys. Rev. Lett. 83, 1719 (1999).

[23] L. Bergstrom, P. Ullio, and J. H. Buckley, Astropart. Phys. 9, 137 (1998).

[24] F. Stoehr, S. D. M. White, V. Springel, G. Tormen, and N. Yoshida, Mon. Not. R. Astron. Soc. 345, 1313 (2003).

[25] L.E. Strigari, S. M. Koushiappas, J. S. Bullock, and M. Kaplinghat, Phys. Rev. D 75, 083526 (2007).

[26] L. Pieri, G. Bertone, and E. Branchini, Mon. Not. R. Astron. Soc. 384, 1627 (2008).

[27] C. Giocoli, L. Pieri, and G. Tormen, Mon. Not. R. Astron. Soc. 387, 689 (2008).

[28] L. Pieri, A. Pizzella, E. M. Corsini, E. Dalla Bontá, and F. Bertola, Astron. Astrophys. 496, 351 (2009).

[29] L. Pieri, M. Lattanzi, and J. Silk, Mon. Not. R. Astron. Soc. 399, 2033 (2009).

[30] E. A. Baltz, B. Berenji, G. Bertone, L. Bergstrom, E. Bloom, T. Bringmann, J. Chiang, J. Cohen-Tanugi et al., J. Cosmol. Astropart. Phys. 07 (2008) 013.

[31] G. D. Martinez, J.S. Bullock, M. Kaplinghat, L.E. Strigari, and R. Trotta, J. Cosmol. Astropart. Phys. 06 (2009) 014.

[32] H.-S. Zhao and J. Silk, Phys. Rev. Lett. 95, 011301 (2005).

[33] G. Bertone, A. R. Zentner, and J. Silk, Phys. Rev. D 72, 103517 (2005).
[34] G. Bertone, Phys. Rev. D 73, 103519 (2006).

[35] M. Fornasa and G. Bertone, Int. J. Mod. Phys. D 17, 1125 (2008).

[36] F. Aharonian et al. (HESS Collaboration), Phys. Rev. D 78, 072008 (2008).

[37] M. Taoso, S.'i. Ando, G. Bertone, and S. Profumo, Phys. Rev. D 79, 043521 (2009).

[38] G. Bertone, M. Fornasa, M. Taoso, and A. R. Zentner, New J. Phys. 11, 105016 (2009).

[39] P. Sandick and S. Watson, Phys. Rev. D 84, 023507 (2011).

[40] D. Merritt, S. Harfst, and G. Bertone, Phys. Rev. D 75, 043517 (2007).

[41] G. Bertone and D. Merritt, Mod. Phys. Lett. A 20, 1021 (2005).

[42] G. Bertone and D. Merritt, Phys. Rev. D 72, 103502 (2005).

[43] L. Pieri, J. Lavalle, G. Bertone, and E. Branchini, Phys. Rev. D 83, 023518 (2011).

[44] R. Catena, N. Fornengo, M. Pato, L. Pieri, and A. Masiero, Phys. Rev. D 81, 123522 (2010).

[45] D. Hooper and T. Linden, Phys. Rev. D 84, 123005 (2011).

[46] A. Morselli and B. Canadas (V.V.o.b.o.t.F.L. collaboration) [Nuovo Cimento Soc. Ital. Fis. C (to be published)], arXiv:1012.2292.

[47] V. Vitale et al. (Fermi-LAT Collaboration), Nucl. Instrum. Methods Phys. Res., Sect. A 630, 147 (2011).

[48] N. Bernal, A. Goudelis, Y. Mambrini, and C. Munoz, J. Cosmol. Astropart. Phys. 01 (2009) 046.

[49] T.F.-L. collaboration, Phys. Rev. Lett. 107, 241302 (2011).

[50] S. Galli, F. Iocco, G. Bertone, and A. Melchiorri, Phys. Rev. D 80, 023505 (2009).

[51] T. R. Slatyer, N. Padmanabhan, and D. P. Finkbeiner, Phys. Rev. D 80, 043526 (2009).

[52] G. Hutsi, J. Chluba, A. Hektor, and M. Raidal [Astron. Astrophys. (to be published)], arXiv:1103.2766.

[53] D. P. Finkbeiner, S. Galli, T. Lin, and T. R. Slatyer [Phys. Rev. D (to be published)], arXiv:1109.6322.

[54] S. Galli, F. Iocco, G. Bertone, and A. Melchiorri, Phys. Rev. D 84, 027302 (2011).

[55] N. Jarosik, C. L. Bennett, J. Dunkley, B. Gold, M. R. Greason, M. Halpern, R.S. Hill, G. Hinshaw et al., Astrophys. J. Suppl. Ser. 192, 14 (2011).

[56] R. Trotta, Contemp. Phys. 49, 71 (2008).

[57] R. Trotta, F. Feroz, M. P. Hobson, L. Roszkowski, and R. Ruiz de Austri, J. High Energy Phys. 12 (2008) 024.

[58] L. Roszkowski, R. Ruiz de Austri, and R. Trotta, Phys. Rev. D 82, 055003 (2010).

[59] R. R. de Austri, R. Trotta, and L. Roszkowski, J. High Energy Phys. 05 (2006) 002.

[60] http://www.superbayes.org/.

[61] F. Feroz, M. P. Hobson, and M. Bridges, Mon. Not. R. Astron. Soc. 398, 1601 (2009).

[62] R. L. Arnowitt, B. Dutta, A. Gurrola, T. Kamon, A. Krislock, and D. Toback, Phys. Rev. Lett. 100, 231802 (2008).

[63] P. J. E. Peebles, Astrophys. J. 153, 1 (1968).

[64] Y. B. Zeldovich, V. G. Kurt, and R. A. Sunyaev, Zh. Eksp. Teor. Fiz. 55, 278 (1968) [J. Exp. Theor. Phys. 28, 146 (1969)].

[65] P. Brun, arXiv:1101.2745. 
[66] T. C. Consortium, arXiv:1008.3703.

[67] P. Brun, E. Moulin, J. Diemand, and J. F. Glicenstein, Phys. Rev. D 83, 015003 (2011).

[68] http://indico.in2p3.fr/contributionDisplay.py?sessionId= $39 \&$ contribId $=88 \&$ confId $=1697$.
[69] K. Bernloehr, AIP Conf. Proc. 1085, 874 (2009).

[70] A. A. Abdo, M. Ackermann, M. Ajello, W. B. Atwood, L. Baldini, J. Ballet, G. Barbiellini, D. Bastieri et al., Astrophys. J. 712, 147 (2010).

[71] G. Gelmini and P. Gondolo, arXiv:1009.3690. 